\title{
The geometry of a positively curved Zoll surface of revolution
}

\author{
By K. Kiyohara, S. V. Sabau *, K. Shibuya
}

\begin{abstract}
In this paper we study the geometry of the manifolds of geodesics of a Zoll surface of positive Gauss curvature, show how these metrics induce Finsler metrics of constant flag curvature and give some explicit constructions.
\end{abstract}

\section{Introduction}

The study of Riemannian manifolds all of whose geodesics are closed has a long history (see [4] for historical remarks). In special, Riemannian manifolds whose geodesics are simple closed curves of equal length, manifolds called today Zoll surfaces, have remarkable geometrical properties extensively studied by many experts (see for instance [4], [6], [5], [7]).

A Zoll surface of revolution $(\Lambda, g)$ is the surface with the local coordinates $(r, \theta) \in$ $[0, \pi] \times \mathbb{R} / 2 \pi \mathbb{Z}$, and the metric

$$
g=[1+h(\cos r)]^{2} d r \otimes d r+\sin ^{2} r d \theta \otimes d \theta,
$$

where $h:[-1,1] \rightarrow(-1,1)$ is an smooth function such that

1. $h(-x)=-h(x)$, for any $x \in[-1,1]$, i.e. it is an odd function,

2. $h(-1)=h(1)=0$.

This is a smooth Riemannian metric on $\mathbb{S}^{2}$ regarded as $(0, \pi) \times \mathbb{R} / 2 \pi \mathbb{Z} \cup\{r=0\} \cup\{r=$ $\pi\}$.

It is known that, the general Zoll metrics near the standard one are parametrized by odd functions $h: \mathbb{S}^{2} \rightarrow \mathbb{R}$, see [4] or [6].

Amongst many other remarkable geometrical properties of Zoll surfaces we mention the fact that the manifold of oriented geodesics $M$ of a Zoll surface $\left(\Lambda=\mathbb{S}^{2}, g\right)$ is a smooth manifold diffeomorphic to $\mathbb{S}^{2}$. It is also known that, for any unit speed geodesic $\gamma$ of $(\Lambda, g)$, the tangent space $T_{[\gamma]} M$ to $M$ in the point $[\gamma]$ is isomorphic to the space of normal Jacobi fields along $\gamma$ (see for instance [4]).

\footnotetext{
${ }^{*}$ Corresponding author
} 
One fundamental question to ask is what kind of natural geometrical structures are carried by the manifold of geodesics $M$ of a Zoll surface $(\Lambda, g)$, and how are these related to the geometry of $(\Lambda, g)$ ?

Some answers are already known. For instance, it is known that $M$ can be endowed with a symplectic structure. Moreover, a Riemannian metric is introduced by Besse ([4], p. 62) on the manifold of geodesics.

In the present paper

- we show that the manifold of geodesics of a positively curved Zoll metric naturally inherits a Finsler structure of constant flag curvature $K=1$.

- Moreover, we construct this Finsler structure and study its geometry. Some examples are also given.

The idea of existence of Finsler metrics of constant flag curvature on the manifold of geodesics of a Zoll metric is not new, originally belonging to Bryant ([3]). However, the concrete construction of such a Finsler metric, its geometrical properties or examples remain unknown until now.

The Finsler metrics of positive constant flag curvature constructed in this paper are intimately related to the original Zoll metric in the sense that the geodesic foliation of the Zoll metric coincides with the indicatrix foliation of the Finsler metric, and the geodesic foliation of the Finsler metric coincides with the indicatrix (that is the unit sphere bundle) foliation of the Zoll metric. All geodesics of a $K=1$ Finsler metric constructed from a positively curved Zoll metric are closed and they intersect each other at a distance $\pi$ from the initial point.

The present paper clarifies the correspondence between a Finsler metric of positive constant flag curvature and a given positively curved Zoll metric. To keep things simple, in the present paper, we restrict ourselves to the simplest case of determining Finsler metrics from Zoll surfaces of revolution, but the more general case of an arbitrary Zoll surface or the higher dimensional case can also be studied. We will consider some of these topics in a forthcoming research.

Here is the structure of our paper.

In Section 2 we recall basic facts about the geometry of Finsler surfaces and its indicatrix (the unit sphere bundle of a Finsler structure). Moreover, we present the peculiarities of Finsler metrics of constant flag curvature $K=1$ and recall the existence theorem of constant sectional curvature Finsler structures due to Bryant (we refer the reader to the original papers [2], 3], or our presentation in [8]).

In Section 3 we recall basic properties of Zoll surfaces which provide the setting necessary to define the geometrical structures on the manifold of geodesics (our main reference here is [4]). Moreover, in order to construct the manifold of geodesics, we explicitly compute the normal Jacobi fields along a geodesic of the Zoll metric, see Proposition 3.1.

Using all these, we move on to the construction of the manifold of geodesics $M$, in Section 4 , by giving the embedding $\iota: \Sigma \rightarrow T M$ of the indicatrix space in the tangent 
space. This is one of our main findings that will lead to the parametric equations of the Finsler indicatrix. Moreover, we introduce local coordinates on the manifold of geodesics in Subsection 4.2.

Now we are able to construct the Finsler metric in Section 5 , by giving the explicit form in coordinates of the parametric equations of the indicatrix curve, see Theorem 5.2 . We also prove that the positivity of the Gauss curvature of the original Zoll metric is in fact equivalent to the positive definiteness of the constant flag curvature Finsler metric in Theorem 5.4. Obviously, in the case $h=0$, the Zoll metric becomes the canonical constant Gauss curvature metric on the sphere and the induced Finsler metric is also the canonical constant Gauss curvature metric on the sphere (see Subsection 5.2.).

In the final section we turn our attention to examples. The most ubiquitous case is when the function $h(x)$ is a polynomial in $x$. In this case the concrete form of the implicit equation of the Finsler indicatrix is given in Theorem 6.2. We apply this result to some concrete examples of positively curved Zoll surfaces constructed by ourselves, see Examples 6.2 and 6.3. We also plot the indicatrix curves of the Finsler surfaces constructed (see Figures 10). We point out that even though we did not write the explicit form of the corresponding Finsler fundamental function $F$, finding the implicit equations of the indicatrices is basically the same thing. Explicitly writing down these fundamental functionsis always possible, but since we have obtain the main geometrical properties of these Finsler metrics, this would not be of much use anyway.

Acknowledgements. We thank to H. Shimada and V. Matveev for many useful discussions.

\section{Finsler surfaces}

\subsection{The geometry of a Finsler surface}

A Finsler norm, or metric, on a real smooth, $n$-dimensional manifold $M$ is a function $F: T M \rightarrow[0, \infty)$ that is positive and smooth on $\widetilde{T M}=T M \backslash\{0\}$, has the homogeneity property $F(x, \lambda v)=\lambda F(x, v)$, for all $\lambda>0$ and all $v \in T_{x} M$, having also the strong convexity property that the Hessian matrix

$$
g_{i j}=\frac{1}{2} \frac{\partial^{2} F^{2}}{\partial y^{i} \partial y^{j}}
$$

is positive definite at any point $u=\left(x^{i}, y^{i}\right) \in \widetilde{T M}$.

The fundamental function $F$ of a Finsler structure $(M, F)$ determines and it is determined by the (tangent) indicatrix, or the total space of the unit tangent bundle of $F$, namely

$$
\Sigma_{F}:=\{u \in T M: F(u)=1\}
$$

which is a smooth hypersurface of $T M$. At each $x \in M$ we also have the indicatrix at $x$

$$
\Sigma_{x}:=\left\{v \in T_{x} M \mid F(x, v)=1\right\}=\Sigma_{F} \cap T_{x} M
$$

which is a smooth, closed, strictly convex hypersurface in $T_{x} M$. 
To give a Finsler structure $(M, F)$ is therefore equivalent to giving a smooth hypersurface $\Sigma \subset T M$ for which the canonical projection $\pi: \Sigma \rightarrow M$ is a surjective submersion and having the property that for each $x \in M$, the $\pi$-fiber $\Sigma_{x}=\pi^{-1}(x)$ is strictly convex including the origin $O_{x} \in T_{x} M$.

In order to study the differential geometry of the Finsler structure $(M, F)$, it is convenient to consider the pull-back bundle $\pi^{*} T M$ with the base manifold $\Sigma$ whose fibers over a point $u \in \Sigma, \pi(u)=x \in M$ are isomorphic to $T_{x} M$ (see [1]).

By defining an orthonormal moving coframing on $\pi^{*} T M$ with respect to the Riemannian metric on $\Sigma$ induced by the Finslerian metric $F$, the moving equations on this frame lead to the so-called Chern connection. This is an almost metric compatible, torsion free connection of the vector bundle $\left(\pi^{*} T M, \pi, \Sigma\right)$.

We are going to restrict ourselves for the rest of the paper to the two dimensional case. To be more precise, our manifold $\Sigma$ will be always 3 -dimensional, and the manifold $M$ will be 2-dimensional, in the case it exists.

It is known (see for instance [1]) that $\Sigma$ becomes a 3-dimensional Riemannian manifold with the metric

$$
\omega^{1} \otimes \omega^{1}+\omega^{2} \otimes \omega^{2}+\omega^{3} \otimes \omega^{3},
$$

that is $\left\{\omega^{1}, \omega^{2}, \omega^{3}\right\}$ is a $g$-orthonormal moving coframe on $\Sigma$. For later use we denote the natural dual basis by $\left\{\hat{e}_{1}, \hat{e}_{2}, \hat{e}_{3}\right\}$.

It is also known that $\left\{\omega^{1}, \omega^{2}, \omega^{3}\right\}$ must satisfy the structure equations

$$
\begin{aligned}
& d \omega^{1}=-I \omega^{1} \wedge \omega^{3}+\omega^{2} \wedge \omega^{3} \\
& d \omega^{2}=\omega^{3} \wedge \omega^{1} \\
& d \omega^{3}=K \omega^{1} \wedge \omega^{2}-J \omega^{1} \wedge \omega^{3},
\end{aligned}
$$

where $I, J, K$ are smooth functions on $\Sigma$ called the invariants of the Finsler structure. More precisely, the functions $I, J, K$ are called the Cartan scalar, the Landsberg curvature and the flag curvature of $(M, F)$, respectively. Equivalently, we have

$$
\begin{aligned}
& {\left[\hat{e}_{1}, \hat{e}_{2}\right]=-K \hat{e}_{3}} \\
& {\left[\hat{e}_{2}, \hat{e}_{3}\right]=-\hat{e}_{1}} \\
& {\left[\hat{e}_{3}, \hat{e}_{1}\right]=-I \hat{e}_{1}-\hat{e}_{2}-J \hat{e}_{3} .}
\end{aligned}
$$

A Finsler surface $(M, F)$ is Riemannian if and only if the Cartan scalar $I$ vanishes everywhere on $\Sigma$.

A very usefull generalization of this notion is the generalized Finsler structure introduced by R. Bryant. In the two dimensional case a generalized Finsler structure is a coframing $\omega=\left(\omega^{1}, \omega^{2}, \omega^{3}\right)$ on a three dimensional manifold $\Sigma$ that satisfies some given structure equations (see [2]). By extension, one can study the generalized Finsler structure $(\Sigma, \omega)$ defined in this way ignoring even the existence of the underlying surface $M$.

Observe that in the case $n>2$, there will be no such globally defined coframing on the $2 n-1$-dimensional manifold $\Sigma$. The reason is that even though the orthonormal frame bundle $\mathcal{F}$ over $M$ does admit a global coframing, it is a peculiarity of the $n=2$ dimensional case that $\mathcal{F}$ can be identified with $\Sigma$ (see also [1], p. 92-93 for concrete computations). 
Definition 2.1 A 3-dimensional manifold $\Sigma$ endowed with a coframing $\omega=\left(\omega^{1}, \omega^{2}, \omega^{3}\right)$ which satisfies the structure equations (2.2) will be therefore called a generalized Finsler surface, where $I, J, K$ are smooth functions on $\Sigma$, called the invariants of the generalized Finsler structure $(\Sigma, \omega)$ (see [2] for details).

As long as we work only with generalized Finsler surfaces, it might be possible that this generalized structure is not realizable as a classical Finslerian structure on a surface $M$. This imposes the following definition [2].

Definition 2.2 A generalized Finsler surface $(\Sigma, \omega)$ is said to be amenable if the leaf space $\mathcal{M}$ of the codimension 2 foliation defined by the equations $\omega^{1}=0, \omega^{2}=0$ is a smooth surface such that the natural projection $\pi: \Sigma \rightarrow \mathcal{M}$ is a smooth submersion.

As R. Bryant emphasizes in [2] the difference between a classical Finsler structure and a generalized one is global in nature, in the sense that every generalized Finsler surface structure is locally diffeomorphic to a classical Finsler surface structure.

The following fundamental result can be also found in [2]

Theorem 2.3 The necessary and sufficient condition for a generalized Finsler surface $(\Sigma, \omega)$ to be realizable as a classical Finsler structure on a surface are

1. the leaves of the foliation $\left\{\omega^{1}=0, \omega^{2}=0\right\}$ are compact;

2. it is amenable, i.e. the space of leaves of the foliation $\left\{\omega^{1}=0, \omega^{2}=0\right\}$ is a differentiable manifold $M$;

3. the canonical immersion $\iota: \Sigma \rightarrow T M$, given by $\iota(u)=\pi_{*, u}\left(\hat{e}_{2}\right)$, is one-to-one on each $\pi$-fiber $\Sigma_{x}$,

where we denote by $\left(\hat{e}_{1}, \hat{e}_{2}, \hat{e}_{3}\right)$ the dual frame of the coframing $\left(\omega^{1}, \omega^{2}, \omega^{3}\right)$.

In the same source it is pointed out that if for example the $\left\{\omega^{1}=0, \omega^{2}=0\right\}$ leaves are not compact, or even in the case they are, if they are ramified, or if the curves $\Sigma_{x}$ winds around origin in $T_{x} M$, in any of these cases, the generalized Finsler surface structure is not realizable as a classical Finsler surface.

An illustrative example found in [2] is the case of an amenable generalized Finsler surface such that the invariant $I$ is constant, however $I$ is not zero. This kind of generalized structure is not realizable as a Finsler surface because $I \neq 0$ means that the leaves of the foliation $\left\{\omega^{1}=0, \omega^{2}=0\right\}$ are not compact. Indeed, in the case $I^{2}<4$, the $\pi$-fibers $\Sigma_{x}$ are logarithmic spirals in $T_{x} M$.

Let us return to the general theory of generalized Finsler structures on surfaces. By taking the exterior derivative of the structure equations (2.2) one obtains the Bianchi equations of the Finsler structure:

$$
J=I_{2}, \quad K_{3}+K I+J_{2}=0,
$$

where we denote by $I_{i}$ the directional derivatives with respect to the coframing $\omega$, i.e. $d f=f_{1} \omega^{1}+f_{2} \omega^{2}+f_{3} \omega^{3}$, for any smooth function $f$ on $\Sigma$. 
Taking now one more exterior derivative of the last formula written above, one obtains the Ricci identities with respect to the generalized Finsler structure

$$
\begin{aligned}
& f_{21}-f_{12}=-K f_{3} \\
& f_{32}-f_{23}=-f_{1} \\
& f_{31}-f_{13}=I f_{1}+f_{2}+J f_{3} .
\end{aligned}
$$

\section{Remarks.}

1. Remark first that the structure equations of a Riemannian surface are obtained from (2.2) by putting $I=J=0$.

2. Since $J=I_{2}$, one can easily see that the necessary and sufficient condition for a generalized Finsler structure to be non-Riemannian is $I \neq 0$.

\subsection{Bryant's existence Theorems}

The existence of Finsler structures of constant flag curvature on the manifold of geodesics of a positively curved Zoll manifold was pointed out for the first time by R. Bryant (see [3]). We will recall in this section Bryant's results and reformulate them in a convenient form for our considerations in the following paragraphs. The theorems in this section are essentially equivalent to the results in 3 .

Observe that in the case $K=1$ the the structure equations 2.2 can be written as

$$
\begin{aligned}
& d \omega^{1}=\left[-I \omega^{1}+\omega^{2}-J \omega^{3}\right] \wedge \omega^{3} \\
& d \omega^{2}=\omega^{3} \wedge \omega^{1} \\
& d \omega^{3}=\omega^{1} \wedge\left[-I \omega^{1}+\omega^{2}-J \omega^{3}\right],
\end{aligned}
$$

with the Bianchi equations

$$
J=I_{2}, \quad I+J_{2}=0,
$$

and that, by using the well-known formula $\mathcal{L}_{X} \omega=i_{X} d \omega+d\left(i_{X} \omega\right)$, we have the following invariance formulas

$$
\begin{aligned}
& \mathcal{L}_{\hat{e}_{2}} \omega^{1}=\omega^{3}, \quad \mathcal{L}_{\hat{e}_{2}} \omega^{2}=0, \quad \mathcal{L}_{\hat{e}_{2}} \omega^{3}=-\omega^{1}, \\
& \mathcal{L}_{\hat{e}_{2}} I=J, \quad \mathcal{L}_{\hat{e}_{2}} J=-I .
\end{aligned}
$$

We observe that in the case of a non-Riemannian Finsler surface with $K=1$, the remaining invariants $I$ and $J$ must be both non-vanishing smooth functions on $\Sigma$.

An elementary computation shows that

$$
\begin{aligned}
& \mathcal{L}_{\hat{e}_{2}}\left[\omega^{1} \otimes \omega^{1}+\omega^{3} \otimes \omega^{3}\right]=0, \quad \mathcal{L}_{\hat{e}_{2}}\left[\omega^{1} \wedge \omega^{3}\right]=0, \\
& \mathcal{L}_{\hat{e}_{2}}\left[I \omega^{1}+J \omega^{3}\right]=0 .
\end{aligned}
$$

We recall that a Finsler structure $(M, F)$ is called geodesically amenable if the set $\Lambda:=\Sigma /\left\langle\hat{e}_{2}\right\rangle=\Sigma /\left\{\omega^{1}=0, \omega^{3}=0\right\}$ of the integral curves of the vector field $\hat{e}_{2}$ can be given a 


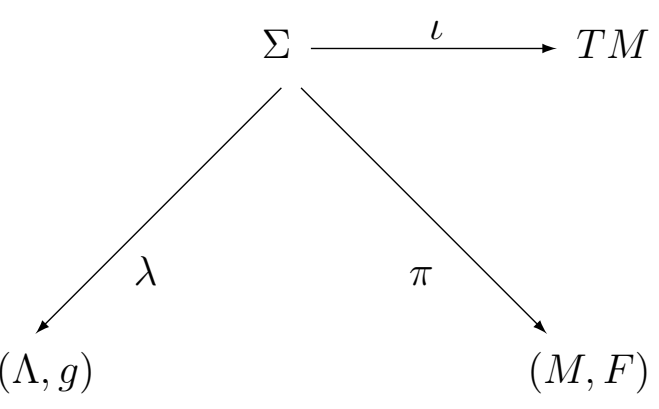

Figure 1: The Cartan double fibration.

structure of smooth manifold of dimension two such that the natural mapping $\lambda: \Sigma \rightarrow \Lambda$ is a smooth submersion (see Figure 1).

In general there is no natural metric on $\Lambda$, but in the case of $K=1$ such a metric does exist.

Theorem 2.4 Let $(M, F)$ be a geodesically amenable Finsler surface with the canonical coframe $\left(\omega^{1}, \omega^{2}, \omega^{3}\right)$ and invariats $I, J$ and $K=1$ on the indicatrix bundle $\Sigma$.

Then, on the manifold of geodesics $\Lambda$ there exists a one-form $\varpi$

$$
\lambda^{*}(\varpi)=I \omega^{1}+J \omega^{3},
$$

and a Riemannian metric $g$ with area form $d A$ and Gauss curvature $G$ determined only by the Finsler structure F. More precisely

$$
\begin{aligned}
& \lambda^{*}(g)=\omega^{1} \otimes \omega^{1}+\omega^{3} \otimes \omega^{3} \\
& \lambda^{*}(d A)=\omega^{1} \wedge \omega^{3}
\end{aligned}
$$

and

$$
\lambda^{*}(G)=1-I^{2}-J^{2}-I_{3}+J_{1} .
$$

Proof. The proof is quite straightforward. From (2.6) it is clear that the one form $I \omega^{1}+J \omega^{3}$ on $\Sigma$ is invariant under the geodesic flow of $F$ and hence it descends on the manifold of geodesics $\Lambda$.

Moreover, by using the equations (2.4) and (2.5) it can be easily shown that the coframe

$$
\alpha^{1}:=\omega^{1}, \alpha^{2}=\omega^{3}, \alpha^{3}=\lambda^{*}(\varpi)-\omega^{2}
$$

gives the Riemannian metric $g$ with sectional curvature $G$ as in the theorem.

Conversely, one has

Theorem 2.5 Let $(\Lambda, g)$ be a geodesically amenable Riemannian surface with the moving coframe $\left(\alpha^{1}, \alpha^{2}, \alpha^{3}\right)$ on the unit sphere bundle $U^{g} \Lambda=\Sigma$ and Gauss curvature $G$, and let us assume that there exists a one form $\varpi$ on $\Lambda$ satisfying the structure equation

$$
d \lambda^{*}(\varpi)=(G-1) \alpha^{1} \wedge \alpha^{2} .
$$


Then, on the manifold of geodesics $M$ there exists a $K=1$ Finsler structure $F$ with the canonical coframe

$$
\omega^{1}=\alpha^{1}, \omega^{2}=\lambda^{*}(\varpi)-\alpha^{3}, \omega^{3}=\alpha^{2},
$$

and the invariants $I=\varpi_{1}, J=\varpi_{2}$, where $\lambda^{*}(\varpi)=\varpi_{1} \alpha^{1}+\varpi_{2} \alpha^{2}$.

The proof is again straightforward using equations (2.4) and (2.5) and the structure equations of a Riemannian structure.

Observe that in the constructions above we always identify the indicatrix bundle $\Sigma$ of the Finsler structure with the unit sphere bundle of the Riemannian metric $g$.

A quick analysis of the leaf quotient spaces shows the following.

1. The $(M, F)$-geodesic foliation $\left\{\omega^{1}=0, \omega^{3}=0\right\}$ coincides with the $(\Lambda, g)$-indicatrix foliation $\left\{\alpha^{1}=0, \alpha^{2}=0\right\}$.

2. The $(M, F)$-indicatrix foliation $\left\{\omega^{1}=0, \omega^{2}=0\right\}$ coincides with the foliation $\left\{\alpha^{1}=\right.$ $\left.0, \alpha^{3}-\lambda^{*}(\varpi)=0\right\}$, called by Bryant, the $\varpi$-foliation on $(\Lambda, g)$.

If we denote by subscripts the directional derivatives, that is $d f=f_{\theta 1} \theta^{1}+f_{\theta 2} \theta^{2}+f_{\theta 3} \theta^{3}$, for any smooth function $f: \Sigma \rightarrow \mathbb{R}$, then one can obtain the following result.

Theorem 2.6 Let $\left(\Lambda=\mathbb{S}^{2}, g\right)$ be a Zoll surface with everywhere positive Gauss curvature $G>0$, and let $M$ be the manifold of oriented geodesics of $g$. Then, there exists a unique Finsler structure with $K=1$ on $M$ with the moving coframe

$$
\begin{aligned}
& \omega^{1}:=\lambda^{*}(\sqrt{G}) \theta^{1} \\
& \omega^{2}:=-\theta^{3} \\
& \omega^{3}:=\lambda^{*}(\sqrt{G}) \theta^{2},
\end{aligned}
$$

where $\left(\theta^{1}, \theta^{2}, \theta^{3}\right)$ is the coframe of $g$.

The invariants of this Finsler structure are given by

$$
I:=\frac{1}{2} \lambda^{*}\left(\frac{G_{\theta 2}}{G^{\frac{3}{2}}}\right), \quad J:=-\frac{1}{2} \lambda^{*}\left(\frac{G_{\theta 1}}{G^{\frac{3}{2}}}\right) .
$$

Proof. The idea is to consider a conformal change of $g$ with a function $u$ and to determine this $u$ by using (2.11).

Indeed, the if we denote by $\left(\alpha^{1}, \alpha^{2}, \alpha^{3}\right)$ the coframe of the conformal metric $u^{2} g$, then

$$
\alpha^{1}=u \theta^{1}, \alpha^{2}=u \theta^{2}, \alpha^{3}=\theta^{3}-* d(\log u),
$$

where $* d(\log u)=-\frac{u_{\theta 2}}{u} \theta^{1}+\frac{u_{\theta 1}}{u} \theta^{2}$.

It results that the coframe (2.12) reads

$$
\omega^{1}=u \theta^{1}, \omega^{2}=\lambda^{*}(\varpi)+* d(\log u)-\theta^{3}, \omega^{3}=u \theta^{2},
$$

where $\lambda^{*}(\varpi)=u\left(I \theta^{1}+J \theta^{2}\right)$. 
An obvious choice is

$$
\lambda^{*}(\varpi)=-* d(\log u)
$$

in which case the coframe 2.15 simplifies to

$$
\omega^{1}=u \theta^{1}, \omega^{2}=-\theta^{3}, \omega^{3}=u \theta^{2} .
$$

Now we can use (2.11) to determine $u$, or equivalently, just to compute the structure equations of the coframe (2.17). However, note that the function $G$ in 2.11) is the curvature of the metric $u^{2} g$. Indeed, observe that $\left(\omega^{1}, \omega^{2}, \omega^{3}\right)$ in (2.17) satisfy the structure equations of a Finsler structure $(2.4)$ if and only if

$$
I=\frac{u_{\theta 2}}{u^{2}}, u^{2}=\lambda^{*}(G), \text { and } J=-\frac{u_{\theta 1}}{u^{2}},
$$

respectively, that is the proof is finished.

Remark 2.7 1. The construction above gives a non-Riemannian Finsler structure of $K=1$ on $M$ if and only if both directional derivatives of $G$ with respect to $\theta^{1}$ and $\theta^{2}$ are non-vanishing functions on $\Lambda$.

2. The Finsler structure constructed in Theorem 2.6 satisfies the extra condition

$$
I_{1}+J_{3}=0
$$

where subscripts are directional derivatives with respect to $\omega^{1}, \omega^{3}$. Indeed, if for an arbitrary smooth function $f$ on $\Sigma$, we denote $d f=f_{1} \omega^{1}+f_{2} \omega^{2}+f_{3} \omega^{3}$, then

$$
f_{\theta^{1}}=\lambda^{*}(\sqrt{G}) f_{1}, f_{\theta^{2}}=\lambda^{*}(\sqrt{G}) f_{3}, f_{\theta^{3}}=-f_{2} .
$$

And hence $I_{\theta 1}+J_{\theta 2}=\sqrt{G}\left(I_{1}+J_{3}\right)$. On the other hand, observe that (2.14) implies $I_{\theta 1}+J_{\theta 2}=0$ and hence $(2.18)$ follows.

Conversely, we can start from a Finsler surface $(M, F)$ of Zoll type on $M=\mathbb{S}^{2}$, that is a Finsler surface all of whose geodesics are closed and have the same length $2 \pi$, with coframe $\left(\omega^{1}, \omega^{2}, \omega^{3}\right)$ on the indicatrix bundle $\Sigma$ and invariants $I, J, K=1$, and construct a Zoll metric on the manifold of geodesics $\Lambda=\mathbb{S}^{2}$.

Indeed, let us consider on $\Sigma$ the directional PDE system with respect to $\left(\omega^{1}, \omega^{2}, \omega^{3}\right)$

$$
\begin{aligned}
& \hat{G}_{1}+2 J \hat{G}=0 \\
& \hat{G}_{3}-2 I \hat{G}=0
\end{aligned}
$$

for an unknown function $\hat{G}: \Sigma \rightarrow \mathbb{R}$. This PDE system has solutions if and only if $I_{1}+J_{3}=0$, and in this case, the solution depends on a constant, only.

More precisely, we need to consider the global existence and the positiveness of the solution. This can be seen as follows. Observe that the condition $I_{1}+J_{3}=0$ implies 
$d\left(-J \omega^{1}+I \omega^{3}\right)=0$, and since $\Sigma$ is diffeomorphic to $\mathbb{R P}^{3}$, the first de Rham cohomology class is zero, hence there exists a function $\hat{\rho}: \Sigma \rightarrow \mathbb{R}$ such that

$$
d \hat{\rho}=-J \omega^{1}+I \omega^{3} .
$$

Since $\hat{e}_{2}(\hat{\rho})=0$ it follows that there exists a function $\rho: \Lambda \rightarrow \mathbb{R}$ such that $\lambda^{*}(\rho)=\hat{\rho}$, and hence the solution we seek will be given by $G=e^{2 \rho}$.

We obtain

Theorem 2.8 Let $\left(M=\mathbb{S}^{2}, F\right)$ be a Finsler surface of Zoll type with coframe $\left(\omega^{1}, \omega^{2}, \omega^{3}\right)$ on the indicatrix bundle $\Sigma$ and invariants $I, J, K=1$, that satisfies the condition $I_{1}+J_{3}=$ 0 .

Then the manifold of geodesics $\Lambda=\mathbb{S}^{2}$ can be endowed with a Riemannian metric $g$ with the coframe

$$
\begin{aligned}
& \theta^{1}=\hat{G}^{-\frac{1}{2}} \omega^{1} \\
& \theta^{2}=\hat{G}^{-\frac{1}{2}} \omega^{3} \\
& \theta^{3}=-\omega^{2}
\end{aligned}
$$

and the Gauss curvature $\hat{G}$, where $\hat{G}$ is the solution of the directional PDE (2.19). In fact $(\Lambda, g)$ is a Zoll manifold.

Indeed, it is clear from our construction that the geodesics of the Riemannian manifold $(\Lambda, g)$ are closed, but the fact that they also have the same length is not proved yet. However, this follows immediately from Wadsley Theorem (see [4], Theorem 7.12, p. 183). This common length can be arranged to be $2 \pi$ by choosing an appropriate constant factor in $\hat{G}$.

Remark 2.9 The Theorems 2.6 and 2.8 are reciprocal each other, leading to the following important question:

There is an one-to-one correspondence between $G>0$ Zoll metrics and $K=1$ Finsler manifolds?

The answer is positive, there is an one-to-one correspondence between $\mathcal{K}$-Cartan structures (in this case $\mathcal{K}=G$ ) and $K=1$ Finsler structures up to diffeomorphism and conformal equivalence (see [9], section 7 for details).

Remark 2.10 Taking into account the invariance formulas (2.6), if we denote by $\xi_{t}$ : $\Sigma \rightarrow \Sigma, t \in \mathbb{R}$, the geodesic flow of $F$, that is the flow of $\hat{e}_{2}$, then it is trivial to see that

$$
\begin{aligned}
& \xi_{t, *}\left(\hat{e}_{1}\right)=\cos t \hat{e}_{1}-\sin t \hat{e}_{3} \\
& \xi_{t, *}\left(\hat{e}_{2}\right)=\hat{e}_{2} \\
& \xi_{t, *}\left(\hat{e}_{3}\right)=\sin t \hat{e}_{1}+\cos t \hat{e}_{3} .
\end{aligned}
$$

It is obvious from the construction presented above that the $K=1$ Finsler structure $F$ induced by a positively curved Zoll metric has all geodesics closed and of same length $2 \pi$. 
Moreover, the unit speed geodesics of this Finsler structure, emanating from a fixed point $p \in M$, intersect at the distance $\pi$ in the same point $q$.

Indeed, let us consider a parametrization $s \mapsto v_{s}$ of the indicatrix $\Sigma_{p} \subset T_{p} M, s \in \mathbb{S}^{1}$, $F\left(v_{s}\right)=1$. It is clear from the general construction that $\hat{e}_{3}$ is tangent to the indicatrix, that is $\frac{d}{d s} v_{s}=-\left.\hat{e}_{3}\right|_{v_{s}}$.

By means of the geodesic flow $\xi_{t}$ introduced above, $\xi_{\pi}\left(v_{s}\right) \in T M$, and moreover we have

$$
\frac{d}{d s} \xi_{\pi}\left(v_{s}\right)=\xi_{\pi, *}\left(\frac{d}{d s} v_{s}\right)=-\left.\hat{e}_{3}\right|_{\xi_{\pi}\left(v_{s}\right)},
$$

where we have used (2.21).

Therefore, there exists a point $q \in M$ such that $\xi_{\pi}\left(v_{s}\right) \in T_{q} M$, for any parameter value $s$, and the statement follows.

\section{The geometry of a Zoll surface of revolution}

In this section we review the basic facts on the geometry of a Zoll surface of revolution needed in the next sections. Our main reference is [4].

\subsection{Geodesics on a Zoll surface}

Let us consider the Zoll surface of revolution $(\Lambda, g)=\left(\mathbb{S}^{2}, g\right)$ with the coordinates and metric $g$ described in Introduction (see (1.1)).

We describe now the geodesics of $(\Lambda, g)$ in terms of the Hamiltonian formalism. We consider the local coordinates $\left(r, \theta ; \xi_{1}, \xi_{2}\right)$ on the cotangent space $T^{*} \Lambda$, and the Hamiltonian function

$$
2 E=\frac{\xi_{1}^{2}}{[1+h(\cos r)]^{2}}+\frac{\xi_{2}^{2}}{\sin ^{2} r} .
$$

Since we have a surface of revolution, $F=\xi_{2}$ is a first integral, i.e. it can be checked by direct computation that the Poisson bracket vanishes $\{E, F\}=0$.

We consider now the geodesics such that $2 E=1$ and $\xi_{2}=c \in[-1,1]$, that is

$$
\left\{\begin{array}{l}
\frac{\xi_{1}^{2}}{[1+h(\cos r)]^{2}}+\frac{\xi_{2}^{2}}{\sin ^{2} r}=1 \\
\xi_{2}=c
\end{array}\right.
$$

It follows

$$
\xi_{1}= \pm[1+h(\cos r)] \sqrt{1-\frac{c^{2}}{\sin ^{2} r}}
$$

We obtain the geodesic (flow) $t \mapsto\left(r(t), \theta(t) ; \xi_{1}(t), \xi_{2}(t)\right)$ given by

$$
\left\{\begin{array}{l}
\frac{d r}{d t}=\frac{\partial E}{\partial \xi_{1}}=\frac{\xi_{1}}{[1+h(\cos r)]^{2}}= \pm \frac{1}{1+h(\cos r)} \sqrt{1-\frac{c^{2}}{\sin ^{2} r}} \\
\frac{d \theta}{d t}=\frac{\partial E}{\partial \xi_{2}}=\frac{\xi_{2}}{\sin ^{2} r}=\frac{c}{\sin ^{2} r} .
\end{array}\right.
$$


Observe that

$$
\frac{d \theta}{d t} \cdot \sin ^{2} r=c
$$

is called the Clairaut constant.

By (3.1) or (3.2) we have $|c| \leq \sin r$. Put

$$
r_{c}=\arcsin c \in[0, \pi / 2] .
$$

Then the range of $r(t)$ along the geodesic is $\left[r_{c}, \pi-r_{c}\right]$. Since $h$ is an odd function, we have

$$
\begin{gathered}
\int_{r_{c}}^{\pi-r_{c}} \frac{d t}{d r}= \pm \int_{r_{c}}^{\pi-r_{c}} \frac{\sin r[1+h(\cos r)]}{\sqrt{\sin ^{2} r-\sin ^{2} r_{c}}} d r= \pm \pi \\
\int_{r_{c}}^{\pi-r_{c}} \frac{d \theta}{d r}= \pm \int_{r_{c}}^{\pi-r_{c}} \frac{\sin r_{c}[1+h(\cos r)]}{\sin r \sqrt{\sin ^{2} r-\sin ^{2} r_{c}}} d r= \pm \pi
\end{gathered}
$$

which indicate that all geodesics are closed and have length $2 \pi$.

Let us now describe the frame $\left(\hat{v}_{1}, \hat{v}_{2}, \hat{v}_{3}\right)$ on the unit tangent bundle $\Sigma=U \Lambda$ by means of the coordinates $(r, \theta)$ on $\Lambda$. To do so, we use the decomposition

$$
T_{v} \Sigma=H_{v}+V_{v} \quad(v \in \Sigma)
$$

of the tangent space into its horizontal and vertical parts and the natural identifications

$$
H_{v} \simeq T_{\lambda(v)} \Lambda, \quad V_{v} \simeq v^{\perp} \subset T_{\lambda(v)} \Lambda .
$$

If $X \in T_{v} \Sigma$ is decomposed to the sum of $X_{1} \in T_{\lambda(v) \Lambda}$ (horizontal part) and $X_{2} \in v^{\perp}$ (vertical part), then we will write it as

$$
X=\left(\begin{array}{l}
X_{1} \\
X_{2}
\end{array}\right)
$$

We define the orientation on $\Lambda$ so that $\partial / \partial r, \partial / \partial \theta$ is positive in this order. Let $n(t)$ be the unit normal vector to $\dot{\gamma}(t)$ such that $\dot{\gamma}(t), n(t)$ is positive in this order. Then they are described as

$$
\begin{aligned}
& \dot{\gamma}(t)=\epsilon \frac{1}{1+h(\cos r)} \sqrt{1-\frac{c^{2}}{\sin ^{2} r}} \frac{\partial}{\partial r}+\frac{c}{\sin ^{2} r} \frac{\partial}{\partial \theta}, \\
& n(t)=\frac{-c}{\sin r[1+h(\cos r)]} \frac{\partial}{\partial r}+\epsilon \frac{\sqrt{\sin ^{2} r-c^{2}}}{\sin ^{2} r} \frac{\partial}{\partial \theta}
\end{aligned}
$$

where $\epsilon= \pm 1$, and the vector fields $\hat{v}_{1}, \hat{v}_{2}, \hat{v}_{3}$ at $\dot{\gamma}(t) \in \Sigma$ are described as follows:

$$
\hat{v}_{1}=\left(\begin{array}{c}
n(t) \\
0
\end{array}\right), \quad \hat{v}_{2}=\left(\begin{array}{c}
\dot{\gamma}(t) \\
0
\end{array}\right), \quad \hat{v}_{3}=\left(\begin{array}{c}
0 \\
n(t)
\end{array}\right) .
$$

For the sake of convinience, we will write $\hat{\gamma}(t)$ instead of $\dot{\gamma}(t)$ when it represents the point of $\Sigma$. Also, we will denote by $[\gamma]$ the corresponding point on the manifold of geodesic, $M$. 


\section{$3.2 \quad$ Jacobi fields}

It is known that the tangent space at a point $\gamma$ to the manifold of geodesics of a Zoll surface is given by the space of Jacobi fields along the geodesic $\gamma$.

We start by decomposing the Killing vector field $\frac{\partial}{\partial \theta}$ along $\gamma(t)$ as

$$
\left.\frac{\partial}{\partial \theta}\right|_{\gamma(t)}=c \cdot \dot{\gamma}(t) \pm \sqrt{\sin ^{2} r-c^{2}} \cdot n(t) .
$$

Let us define the Jacobi field

$$
Y(t):=y(t) n(t), \quad y(t)= \pm \sqrt{\sin ^{2} r(t)-c^{2}}
$$

along $\gamma(t)$, and compute

$$
y^{\prime}(t)=\frac{\cos r}{1+h(\cos r)}
$$

Observe that

$$
Y(0)=0 \text {, i.e. } y(0)=0 \text {. }
$$

If we take one more derivative, we can compute the Gauss curvature $G(r)$ of this Zoll metric by means of the Jacobi equation, that is

$$
y^{\prime \prime}(t)=\mp\left[1-\frac{\cos r h^{\prime}(\cos r)}{1+h(\cos r)}\right] \frac{\sqrt{\sin ^{2} r-c^{2}}}{[1+h(\cos r)]^{2}}=-G(\gamma(t)) y(t),
$$

where

$$
\hat{G}(r)=G(r)=\frac{1}{[1+h(\cos r)]^{3}}\left[1+h(\cos r)-\cos r \cdot h^{\prime}(\cos r)\right],
$$

that is usual formula for the Gauss curvature of a Zoll metric (see for instance [4], p. 105).

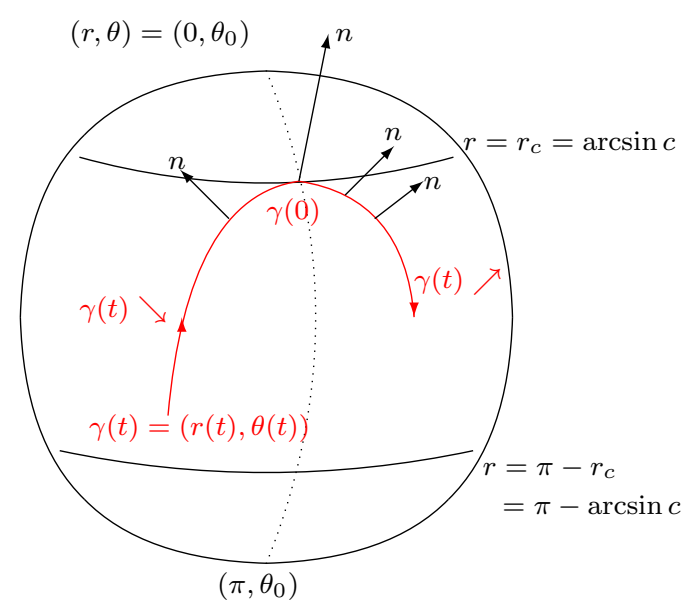

Figure 2: A geodesic $\gamma$ on the Zoll sphere. 
Let us consider the parameter $t$ on the geodesic $\gamma$ such that at $t=0, \gamma(0)=r_{c}:=$ $\arcsin c$, see Figure 2 .

We will construct in the following two normalized Jacobi fields $Y_{1}$ and $Y_{2}$ along $\gamma(t)$. Here normalized means that we will determine these Jacobi fields subject to the initial conditions

$$
\left\{\begin{array}{l}
Y_{1}(0)=0, \quad Y_{1}^{\prime}(0)=n(0) \\
Y_{2}(0)=n(0), \quad Y_{2}^{\prime}(0)=0 .
\end{array}\right.
$$

Proposition 3.1 The normalized Jacobi fields along $\gamma(t)$ are given by $Y_{i}(t):=y_{i}(t) n(t)$, $i=1,2$, where

$$
\begin{aligned}
& y_{1}(t)=\frac{1+h\left(\cos r_{c}\right)}{\cos r_{c}} \cdot y(t)= \pm \frac{1+h\left(\cos r_{c}\right)}{\cos r_{c}} \sqrt{\sin ^{2} r-c^{2}} \\
& y_{2}(t)=\frac{1}{y_{1}^{\prime}(t)}-y_{1}(t) \int_{0}^{t} \frac{K(\gamma(s))}{\left[y_{1}^{\prime}(s)\right]^{2}} d s
\end{aligned}
$$

Proof. We can check by direct computation that these $Y_{i}(t)$ satisfy the initial conditions (3.12) by using the computation for $y(t)$.

\section{The manifold of geodesics}

\subsection{The embedding of $\Sigma$ into the tangent space $T M$}

To define the desired Finsler metric on $M$, we will embed $\Sigma$ into $T M$ so that $\Sigma$ is realized as the indicatrix bundle. Since the vector field $\hat{e}_{2}$ will be the generator of the geodesic flow, this embedding should be given by (compare with Bryant [3])

$$
\iota: \Sigma \rightarrow T M, \quad u \rightarrow \iota(u)=\pi_{*, u}\left(\hat{e}_{2}\right) .
$$

Let us describe

$$
\iota(\hat{\gamma}(t))=a(t) Y_{1}+b(t) Y_{2} \in T_{[\gamma]} M,
$$

where $Y_{i}$ are the tangent vectors to $M$ at $[\gamma]$ which correspond to the Jacobi fields $Y_{i}(t)$ along $\gamma(t)$ defined before. One obtains:

Proposition 4.1 $a(t)=-y_{2}(t), \quad b(t)=y_{1}(t)$.

Proof. $\quad$ Since $\hat{e}_{2}=-\hat{v}_{3}$, we have

$$
a(t)\left(\begin{array}{c}
Y_{1}(t) \\
Y_{1}^{\prime}(t)
\end{array}\right)+b(t)\left(\begin{array}{c}
Y_{2}(t) \\
Y_{2}^{\prime}(t)
\end{array}\right)=-\left(\begin{array}{c}
0 \\
n(t)
\end{array}\right),
$$

that is, in scalar form,

$$
a(t)\left(\begin{array}{l}
y_{1}(t) \\
y_{1}^{\prime}(t)
\end{array}\right)+b(t)\left(\begin{array}{l}
y_{2}(t) \\
y_{2}^{\prime}(t)
\end{array}\right)=-\left(\begin{array}{l}
0 \\
1
\end{array}\right) .
$$

Thus the proposition follws. 


\subsection{Coordinates on the manifold of geodesics}

In this section we introduce a system of coordinates

$$
(R, \Theta) \in\left(-\frac{\pi}{2}, \frac{\pi}{2}\right) \times \mathbb{R} / 2 \pi \mathbb{Z}
$$

on the manifold of geodesics $M$, which is a 2-dimensional manifold, as follows.

The base manifold of the Zoll surface $(\Lambda, g)$ is $\mathbb{S}^{2}$ with the usual spherical coordinates $(r, \theta) \in[0, \pi] \times \mathbb{R} / 2 \pi \mathbb{Z}$. However, in the present context we will consider negative values of the coordinate $r$ as well, that is, for a fixed $\theta=\theta_{0}$, the half meridian $\left\{(r, \theta):-\frac{\pi}{2}<\right.$ $\left.r<\frac{\pi}{2}, \theta=\theta_{0}\right\}$ is included in the northern hemisphere, see Figure 3 .

If we consider a unit length tangent vector in the direction $\frac{\sigma}{\partial \theta}$ at $\left(r_{0}, \theta_{0}\right)$, for some $0<r_{0}<\frac{\pi}{2}$, it is easy to see that this tangent vector can be smoothly extended to a vector field on $\Lambda$ for any $-\frac{\pi}{2}<r \leq 0$.

In other words, for the same non-oriented geodesic $\gamma_{0}$ with initial conditions $\gamma_{0}(0)=$ $\left(r_{0}, \theta_{0}\right), \dot{\gamma}(0)=\frac{1}{\sin r_{0}}$, we need to make difference if the initial velocity is in the direction of $\frac{\partial}{\partial \theta}$ or opposite direction. Observe that in terms of Clairaut constants, these geodesics correspond to $c_{0}>0$ and $c_{0}<0$, and therefore we will denote these oriented geodesics by $\gamma_{0}^{+}$and $\gamma_{0}^{-}$, respectively. On the manifold of oriented geodesics $M$ they will give different points $\left[\gamma_{0}^{+}\right]$and $\left[\gamma_{0}^{-}\right]$.

In order to introduce local coordinates on the manifold of geodesics, it is usefull to make the following convention. In order to make distinction between $\gamma_{0}^{+}$and $\gamma_{0}^{-}$, we will identify $\gamma_{0}^{-}$with the geodesic on $(\Lambda, g)$ with initial point $\left(-r_{0}, \theta+\pi\right)$ and Clairaut constant $-c_{0}$.



Figure 3: Coordinates on the Zoll sphere.

From topological reasons it is known that the manifold of geodesics is a sphere, so we will start by introducing the local coordinates $(R, \Theta)$ on $M=\mathbb{S}^{2}$ except two points, the poles on $M$, that correspond to the two equators $\left\{r=\frac{\pi}{2}\right\}$ of different orientations $\varepsilon^{+}$and $\varepsilon^{-}$, that is geodesics with Clairaut constants $c= \pm 1$.

For a geodesic $\gamma(t)=(r(t), \theta(t))$ of $(\Lambda, g)$, with Clairaut constant $c \neq \pm 1$, we define 
the local coordinates $(R, \Theta)$ of the corresponding point $[\gamma] \in M$ as follows

$$
(R, \Theta)=\left\{\begin{array}{l}
(r(0), \theta(0)), \text { if } c>0 \\
(-r(0), \theta(0)+\pi), \text { if } c<0 \\
\left(0, \theta(\dot{\gamma}(0))-\frac{\pi}{2}\right), \text { if } c=0
\end{array}\right.
$$

(see figures 4, 5).

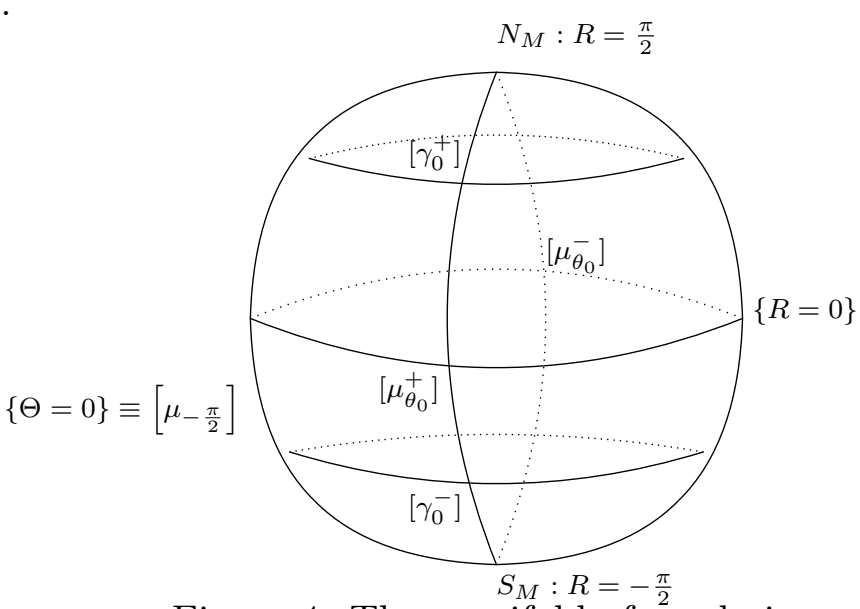

Figure 4: The manifold of geodesics.

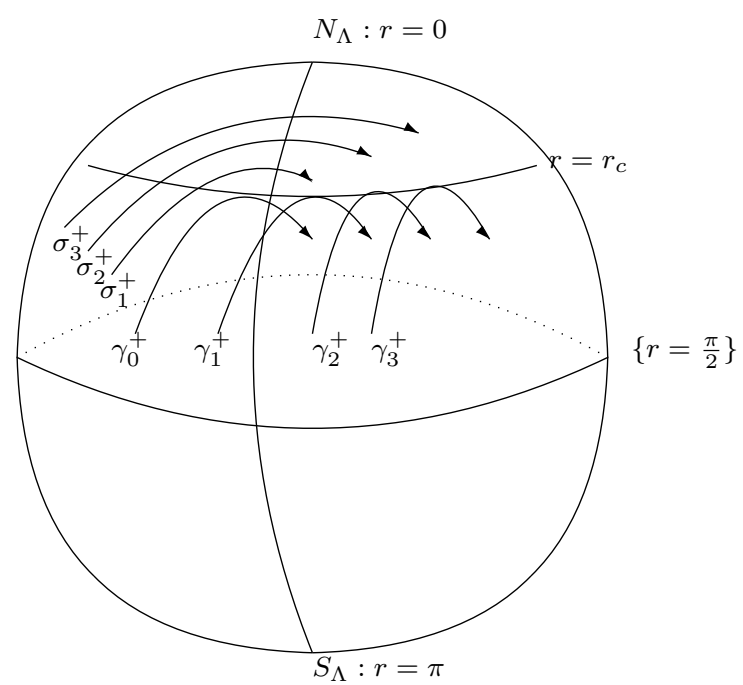

Figure 5: A geodesic variation on the Zoll sphere.

Remark 4.2 By considering a geodesic variation of the base geodesic $\gamma_{0}^{+}$with the variation vector fields $Y_{1}$ and $Y_{2}$, we obtain the variations $\gamma_{s}^{+}$and $\sigma_{s}^{+}$,respectively. By the coordinates system above they will give points on the parallel $R=r_{c}$ and the meridian $\Theta=\theta_{0}$.

Remark 4.3 Let us consider geodesics on $(\Lambda, g)$ that start from the Nothern Pole $p_{0}:=$ $N_{\Lambda}$, that is meridians. Let us also consider an orthonormal basis $v_{0}, v_{1}$ of $T_{p_{0}} \Lambda$ positive 
oriented (we use here $\mathbb{S}^{2}$ orientation) defined as follows. For a geodesic $\gamma_{\theta}^{+}(t)=(r(t), \theta)$, with $\theta$ constant, obtained from the variation of $\gamma_{0}^{+}(t)=(r(t), 0), t \in(0, \pi)$, with variation vector field $Y_{2}$ passing through the pole $p_{0}$, that is a meridian, we have $\gamma_{\theta}^{+}(0)=p_{0}$. We put

$$
\dot{\gamma}_{\theta}(0)=\cos \theta \cdot v_{0}+\sin \theta \cdot v_{1} .
$$

In order to induce a smooth manifold structure on $M$, we identify the geodesic from $p_{0}$ and initial velocity 4.2 with the geodesic with same initial point and initial velocity $J\left(\dot{\gamma}_{\theta}(0)\right)$, where $J: T_{p} \Lambda \rightarrow T_{p} \Lambda$ is linear mapping that gives the positive rotation by $\frac{\pi}{2}$ in any tangent plane to a point of $\Lambda$ (see Figure 7, 8).

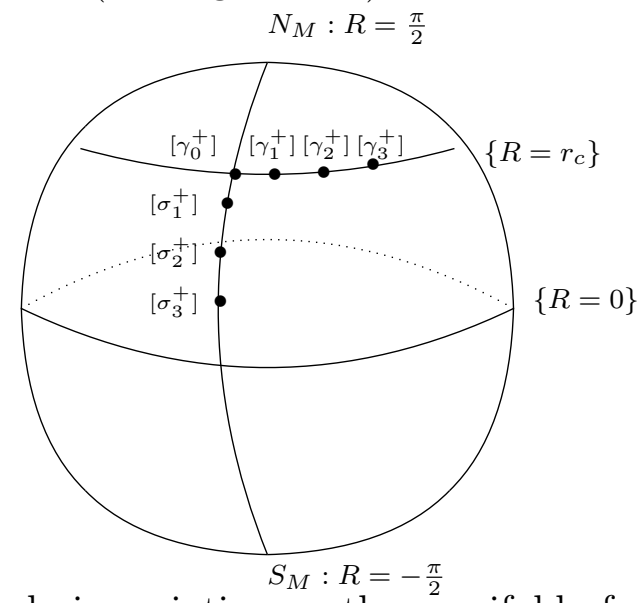

Figure 6: A geodesic variation on the manifold of geodesics.

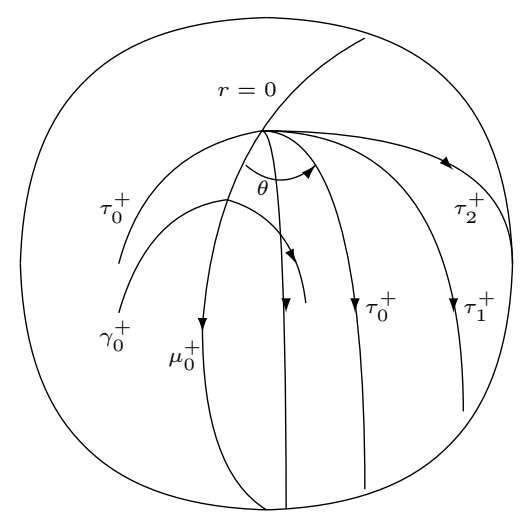

Figure 7: Meridians on the Zoll sphere.

\section{The Finsler metric on the manifold of geodesics}

\subsection{The Finslerian indicatrix}

We will express the Jacobi vector fields $Y_{1}, Y_{2}$ in the canonical coordinates of $T M$, that is 


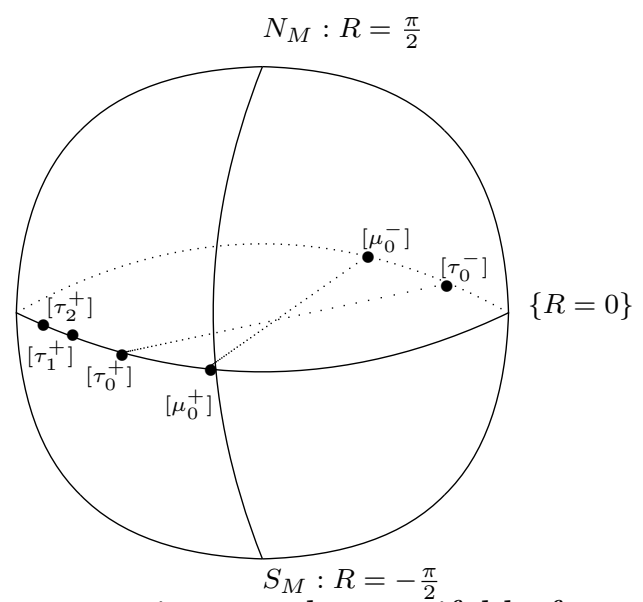

Figure 8: Equator points on the manifold of geodesics.

Proposition 5.1 We have

$$
\begin{aligned}
Y_{1} & =c_{1} \frac{\partial}{\partial \Theta} \\
Y_{2} & =\frac{1}{c_{1} \cos r_{c}} \frac{\partial}{\partial R},
\end{aligned}
$$

where $c_{1}:=\frac{1+h\left(\cos r_{c}\right)}{\cos r_{c}}, r_{c}=\arcsin c, 0<c<\frac{\pi}{2}$. Moreover

$$
\begin{aligned}
& y_{1}^{\prime}=c_{1} \frac{\cos r}{1+h(\cos r)} \\
& y_{2}^{\prime}= \pm \frac{1}{c_{1}} \frac{\cos r}{1+h(\cos r)} \int_{r_{c}}^{r} \frac{\sin s}{\cos ^{2} s}\left[1-\frac{\cos s \cdot h^{\prime}(\cos s)}{1+h(\cos s)}\right]\left[\frac{1+h(\cos s)}{\sqrt{\sin ^{2} s-c^{2}}}\right] d s,
\end{aligned}
$$

where $R \leq r \leq \pi-R, 0<R<\frac{\pi}{2}$, and the sign \pm is the sign of $\dot{r}(t)$.

Proof. Recall that by definition $Y_{1}(t)=c_{1} Y(t)$, where $Y(t)$ is the normal component of $\left.\frac{\partial}{\partial \theta}\right|_{\gamma(t)}$, hence by the definition of our local coordinates on $M$, first formula in (5.1) follows immediately, by identifying $Y(t)$ with $\frac{\partial}{\partial \Theta}$.

Likewise, if we observe that the unit length vector field in the $\left.\frac{\partial}{\partial r}\right|_{\gamma(0)}$ direction is

$$
\left.\frac{1}{\left\|\left.\frac{\partial}{\partial r}\right|_{\gamma(0)}\right\|} \frac{\partial}{\partial r}\right|_{\gamma(0)}=\left.\frac{1}{1+h\left(\cos r_{c}\right)} \frac{\partial}{\partial r}\right|_{\gamma(0)}
$$

we obtain the second formula in (5.1).

For the second set of formulas, observe that by definition $y_{1}(t)=c_{1} y(t)$, that is $y_{1}^{\prime}(t)=c_{1} y^{\prime}(t)$ and using (3.8) the first formula in (5.2) follows immediately.

Next, we start by taking the derivative of $y_{2}(t)$ from (3.13), that is

$$
y_{2}^{\prime}(t)=\frac{d}{d t}\left[\frac{1}{y_{1}^{\prime}(t)}\right]-y_{1}^{\prime}(t) \int_{0}^{t} \frac{G(\gamma(s))}{\left[y_{1}^{\prime}(s)\right]^{2}} d s-y_{1}(t) \frac{d}{d t} \int_{0}^{t} \frac{G(\gamma(s))}{\left[y_{1}^{\prime}(s)\right]^{2}} d s,
$$

and using Leibnitz chain formula and the Jacobi equation it results

$$
y_{2}^{\prime}(t)=-y_{1}^{\prime}(t) \int_{0}^{t} \frac{G(\gamma(s))}{\left[y_{1}^{\prime}(s)\right]^{2}} d s .
$$


By using formula for $y_{1}^{\prime}(t)$, the expression of the Gauss curvature (3.11), and (3.1), the desired formula follows immediately after changing the variable from curve parameter $t$ to $r$ due to the obvious relation

$$
\int_{0}^{t} \frac{G(\gamma(s))}{\left[y_{1}^{\prime}(s)\right]^{2}} d s=\frac{1}{c_{1}^{2}} \int_{r_{c}}^{r} \frac{\sin s}{\cos ^{2} s}\left[1-\frac{\cos s \cdot h^{\prime}(\cos s)}{1+h(\cos s)}\right]\left[ \pm \frac{1+h(\cos s)}{\sqrt{\sin ^{2} s-c^{2}}}\right] d s
$$

We recall that due to the local coordinates definition, we have $c=\sin R, R=r_{c}$.

Observe that the embedding $\iota: \Sigma \rightarrow T M$ reads now

$$
\iota(\hat{\gamma}(t))=-c_{1} y_{2}(t) \frac{\partial}{\partial \Theta}+\frac{y_{1}(t)}{1+h(\cos R)} \frac{\partial}{\partial R},
$$

that is, if we denote by $\left(R, \Theta ; v_{1}, v_{2}\right)$ the canonical coordinates on $T M$,

$$
\iota(\hat{\gamma}(t))=v_{1}(r) \frac{\partial}{\partial R}+v_{2}(r) \frac{\partial}{\partial \Theta},
$$

hence the geodesic flow parametric equations read

$$
v_{1}(r)=\frac{y_{1}(t)}{1+h(\cos R)}= \pm \frac{1}{\cos R} \cdot \sqrt{\sin ^{2} r-c^{2}}
$$

and

$$
\begin{aligned}
v_{2}(r) & =-c_{1} y_{2}(t)=-c_{1} \frac{1}{y_{1}^{\prime}(t)}+c_{1} y_{1}(t) \int_{0}^{t} \frac{G(\gamma(s))}{\left[y_{1}^{\prime}(s)\right]^{2}} d s \\
& =-\frac{1+h(\cos r)}{\cos r} \pm c_{1} \frac{1+h\left(\cos r_{c}\right)}{\cos r_{c}} \sqrt{\sin ^{2} r-c^{2}} \int_{0}^{t} \frac{G(\gamma(s))}{\left[y_{1}^{\prime}(s)\right]^{2}} d s \\
& =-\frac{1+h(\cos r)}{\cos r}+\sqrt{\sin ^{2} r-c^{2}} \int_{r_{c}}^{r} \frac{\sin s}{\cos ^{2} s}\left[1-\frac{\cos s \cdot h^{\prime}(\cos s)}{1+h(\cos s)}\right]\left[ \pm \frac{1+h(\cos s)}{\sqrt{\sin ^{2} s-c^{2}}}\right] d s
\end{aligned}
$$

where we have used definition formula for $c_{1},(3.13)$ and (5.3).

Hence, we get

Theorem 5.2 The parametric equations of the corresponding Finsler metric of constant flag curvature $K=1$ are

$$
\begin{aligned}
& v_{1}(r)= \pm \frac{1}{\cos R} \cdot \sqrt{\sin ^{2} r-c^{2}} \\
& v_{2}(r)=-\frac{1+h(\cos r)}{\cos r}+\sqrt{\sin ^{2} r-c^{2}} \int_{r_{c}}^{r} \frac{\sin s}{\cos ^{2} s}\left[1-\frac{\cos s \cdot h^{\prime}(\cos s)}{1+h(\cos s)}\right]\left[\frac{1+h(\cos s)}{\sqrt{\sin ^{2} s-c^{2}}}\right] d s .
\end{aligned}
$$

Remark 5.3 Using the equality

$$
\int \frac{\sin s}{\cos ^{2} s} \frac{1}{\sqrt{\sin ^{2}-c^{2}}} d s=\frac{1}{1-c^{2}} \frac{\sqrt{\sin ^{2} s-c^{2}}}{\cos s}+\text { constant }
$$


one can rewrite $v_{2}$ as

$$
\begin{gathered}
v_{2}=-[1+h(\cos r)] \frac{\cos r}{\cos ^{2} R}-\frac{\sin ^{2} r-c^{2}}{\cos ^{2} R} h^{\prime}(\cos r) \\
-\frac{\sqrt{\sin ^{2} r-c^{2}}}{\cos ^{2} R} \int_{R}^{r} \sin s \sqrt{\sin ^{2} s-c^{2}} h^{\prime \prime}(\cos s) d s,
\end{gathered}
$$

which eliminates the apparent singularity at $r=\pi / 2$ in the former expression.

Theorem 5.4 The strong convexity of the Finsler indicatrix (5.5) is equivalent to the curvature condition $G>0$ of the Zoll metric.

Proof. Indeed, observe that we have

$$
\begin{aligned}
& v_{1}(r)=\frac{1}{1+h(\cos R)} y_{1}(t) \\
& \dot{v}_{1}(r)=\frac{1}{1+h(\cos R)} y_{1}^{\prime}(t) \frac{d t}{d r} \\
& \ddot{v}_{1}(r)=\frac{1}{1+h(\cos R)}\left[y_{1}^{\prime \prime}(t)\left(\frac{d t}{d r}\right)^{2}+y_{1}^{\prime}(t) \frac{d^{2} t}{d r^{2}}\right]
\end{aligned}
$$

and

$$
\begin{aligned}
& v_{2}(r)=-\frac{1+h(\cos R)}{\cos R} y_{2}(t) \\
& \dot{v}_{2}(r)=-\frac{1+h(\cos R)}{\cos R} y_{2}^{\prime}(t) \frac{d t}{d r} \\
& \ddot{v}_{2}(r)=-\frac{1+h(\cos R)}{\cos R}\left[y_{2}^{\prime \prime}(t)\left(\frac{d t}{d r}\right)^{2}+y_{2}^{\prime}(t) \frac{d^{2} t}{d r^{2}}\right]
\end{aligned}
$$

where $\dot{v}_{i}$ and $\ddot{v}_{i}$ are the first and second derivative of $v_{i}(r)$ with respect to $r$, for $i \in\{1,2\}$.

We can compute now the curvature $k(r)$ of the indicatrix curve $\left(v_{1}(r), v_{2}(r)\right)$, and by using the Jacobi equation, we get

$$
k(r):=\frac{\ddot{v}_{1} \dot{v}_{2}-\ddot{v}_{2} \dot{v}_{1}}{\dot{v}_{1} v_{2}-\dot{v}_{2} v_{1}}=\left(\frac{d t}{d r}\right)^{2} G,
$$

hence the conclusion follows from our construction.

\subsection{The round sphere case $h=0$}

The trivial example of a Zoll sphere of revolution is the canonical Riemannian sphere given by $h=0$. In this case, the indicatrix equations read

$$
\begin{aligned}
& v_{1}(r)=\frac{1}{\cos R} \cdot \sqrt{\sin ^{2} r-c^{2}} \\
& v_{2}(r)=-\frac{1}{\cos r}+\frac{1}{\cos ^{2} R} \frac{\sin ^{2} r-c^{2}}{\cos r}=\frac{\sin ^{2} r+\cos ^{2} R-c^{2}}{\cos ^{2} R \cos r}=\frac{\sin ^{2} r-1}{\cos ^{2} R \cos r}=-\frac{\cos r}{\cos ^{2} R} .
\end{aligned}
$$


Observe that this is equivalent to

$$
\begin{aligned}
& \sin ^{2} r=v_{1}^{2} \cos ^{2} R+c^{2} \\
& \cos ^{2} r=v_{2}^{2} \cos ^{4} R,
\end{aligned}
$$

and by adding these relations we get

$$
v_{1}^{2}+\cos ^{2} R \cdot v_{2}^{2}=1,
$$

where we have used $1-c^{2}=\cos ^{2} R$.

It is clear that this is an ellipse in $T_{p} M$ corresponding to the Riemannian canonical sphere, therefore we have

Proposition 5.5 In the case $h=0$, the corresponding metrical structure on $M$ is the canonical Riemannian sphere.

Remark 5.6 Observe that our local coordinates on $M$ are $(R, \Theta) \in\left[-\frac{\pi}{2}, \frac{\pi}{2}\right] \times \mathbb{R} / 2 \pi \mathcal{Z}$, and that by the coordinates changing $\widetilde{R}:=R+\frac{\pi}{2}$ we obtain $\widetilde{R} \in[0, \pi]$ and hence $(5.9)$ becomes $v_{1}^{2}+\sin ^{2} R \cdot v_{2}^{2}=1$, that is the expected ellipse in the case of the canonical Riemannian sphere.

\section{Examples}

\subsection{The case when $h$ is polynomial}

Let us consider the case when the function $x \mapsto h$ is a polynomial of odd order, that is

$$
h(x)=\sum_{k=0}^{n} a_{2 k+1} x^{2 k+1},
$$

where $n \geq 1$, i.e. the degree of $h$ is greater than two, provided $G>0$, and $\sum_{k=0}^{n} a_{2 k+1}=0$, with $G$ given in (3.11). It can be seen that this function $h$ satisfies the conditions needed to induce a Zoll metric on $\mathbb{S}^{2}$, see Section 3 .

Observe that

$$
h^{\prime}(x)=\sum_{k=0}^{n}(2 k+1) a_{2 k+1} x^{2 k}
$$

and

$$
h^{\prime \prime}(x)=\sum_{k=1}^{n} 2 k(2 k+1) a_{2 k+1} x^{2 k-1}=\sum_{k=0}^{n-1} b_{2 k+1} x^{2 k+1},
$$

where we put $b_{2 k+1}:=2(k+1)(2 k+3) a_{2 k+3}$, for all integers $k \in\{0, \ldots, n-1\}$.

Lemma 6.1 For a real number $\lambda \geq x$ and any integer $k \in\{0, \ldots, n-1\}$, we have

$$
\int \sqrt{\lambda^{2}-x^{2}} x^{2 k+1} d x=\lambda^{2 k}\left(\lambda^{2}-x^{2}\right)^{3 / 2} \sum_{\rho=0}^{k} \frac{(-1)^{\rho+1}}{(2 \rho+3) \lambda^{2 \rho}}\left(\begin{array}{c}
k \\
\rho
\end{array}\right)\left(\lambda^{2}-x^{2}\right)^{\rho}+\mathcal{C},
$$

where $\mathcal{C}$ is arbitrary constant. 
Indeed, if we use the substitution $u:=\lambda^{2}-x^{2}$, it follows

$$
x^{2 k}=\left(\lambda^{2}-u\right)^{k}=\sum_{\rho=0}^{k}(-1)^{\rho}\left(\begin{array}{c}
k \\
\rho
\end{array}\right) \lambda^{2(k-\rho)} u^{\rho},
$$

and hence, we have

$$
\begin{aligned}
& \int \sqrt{\lambda^{2}-x^{2}} x^{2 k+1} d x=-\frac{1}{2} \int \sqrt{u}\left(\lambda^{2}-u\right)^{k} d u \\
& =\frac{1}{2} \sum_{\rho=0}^{k}(-1)^{\rho+1}\left(\begin{array}{c}
k \\
\rho
\end{array}\right) \lambda^{2(k-\rho)} \int \sqrt{u} u^{\rho} d u \\
& =\lambda^{2 k} \sum_{\rho=0}^{k} \frac{(-1)^{\rho+1}}{(2 \rho+3) \lambda^{2 \rho}}\left(\begin{array}{c}
k \\
\rho
\end{array}\right)\left(\lambda^{2}-x^{2}\right)^{\rho+3 / 2}+\mathcal{C},
\end{aligned}
$$

where $\mathcal{C}$ is arbitrary constant.

Next, we compute

$$
\begin{aligned}
& \int \sqrt{\lambda^{2}-x^{2}} h^{\prime \prime}(x) d x=\sum_{k=0}^{n-1} b_{2 k+1} \int \sqrt{\lambda^{2}-x^{2}} x^{2 k+1} d x \\
& =\left(\lambda^{2}-x^{2}\right)^{3 / 2} \sum_{k=0}^{n-1} b_{2 k+1} \lambda^{2 k} \sum_{\rho=0}^{k} \frac{(-1)^{\rho+1}}{(2 \rho+3) \lambda^{2 \rho}}\left(\begin{array}{c}
k \\
\rho
\end{array}\right)\left(\lambda^{2}-x^{2}\right)^{\rho}+\mathcal{C},
\end{aligned}
$$

where $\mathcal{C}$ is an arbitrary constant.

Moreover, we have

$$
\int_{R}^{r} \sin s \sqrt{\sin ^{2} s-c^{2}} h^{\prime \prime}(\cos s) d s=-\int_{\cos R}^{\cos r} \sqrt{\cos ^{2} R-x^{2}} h^{\prime \prime}(x) d x,
$$

where we have used $c=\sin R$ and the substitution $x=\cos s$.

It follows

$$
\begin{aligned}
& \int_{R}^{r} \sin s \sqrt{\sin ^{2} s-c^{2}} h^{\prime \prime}(\cos s) d s \\
& =\left(\cos ^{2} R-\cos ^{2} r\right)^{3 / 2} \sum_{k=0}^{n-1} b_{2 k+1} \cos ^{2 k} R \sum_{\rho=0}^{k} \frac{(-1)^{\rho}}{(2 \rho+3) \cos ^{2 \rho} R}\left(\begin{array}{c}
k \\
\rho
\end{array}\right)\left(\cos ^{2} R-\cos ^{2} r\right)^{\rho} \\
& =\cos ^{3} R v_{1}^{3} \sum_{k=0}^{n-1} c_{2 k+1} \sum_{\rho=0}^{k} d_{\rho} v_{1}^{2 \rho}
\end{aligned}
$$

where we use $\sin ^{2} r-c^{2}=\cos ^{2} R-\cos ^{2} r=\cos ^{2} R v_{1}^{2}$, and put $c_{2 k+1}:=b_{2 k+1} \cos ^{2 k} R$, and $d_{\rho}:=\frac{(-1)^{\rho}}{(2 \rho+3)}\left(\begin{array}{l}k \\ \rho\end{array}\right)$. 
On the other hand, observe that

$$
\begin{aligned}
\frac{1+h(x)}{\cos ^{2} R} x \frac{\cos ^{2} R-x^{2}}{\cos ^{2} R} h^{\prime}(x) & =\frac{x}{\cos ^{2} R}+\frac{1}{\cos ^{2} R} \sum_{k=0}^{n} a_{2 k+1}\left[(2 k+1) \cos ^{2} R-2 k x^{2}\right] x^{2 k} \\
& = \pm \frac{\sqrt{1-v_{1}^{2}}}{\cos R}+\sum_{k=0}^{n} a_{2 k+1} \cos ^{2 k} R\left(1+2 k v_{1}^{2}\right)\left(1-v_{1}^{2}\right)^{k}
\end{aligned}
$$

where we have used $x:=\cos r$ and hence $x^{2}=\cos ^{2} R\left(1-v_{1}^{2}\right)$.

We obtain

Theorem 6.2 In the case when $h$ is the odd polynomial of degree $2 n+1$ given in (6.1) satisfying $G>0$, then the implicit equation of the Finsler indicatrix is

$$
\begin{aligned}
v_{2} & = \pm \frac{\sqrt{1-v_{1}^{2}}}{\cos R}-\sum_{k=0}^{n} a_{2 k+1} \cos ^{2 k} R\left(1+2 k v_{1}^{2}\right)\left(1-v_{1}^{2}\right)^{k} \\
& \pm \cos ^{2} R v_{1}^{4} \sum_{k=0}^{n-1} b_{2 k+1} \cos ^{2 k} R \sum_{\rho=0}^{k} \frac{(-1)^{\rho}}{(2 \rho+3)}\left(\begin{array}{c}
k \\
\rho
\end{array}\right) v_{1}^{2 \rho}
\end{aligned}
$$

where we put $b_{2 k+1}:=2(k+1)(2 k+3) a_{2 k+3}$, for all integers $k \in\{0, \ldots, n-1\}$.

We remark that the sums in the right hand side looks like $2 n+2$-polynomials, but it is easy to see that actually they are only $2 n$-degree polynomials. We give here only the general form.

\subsection{Example 1. The case $h(x)=\varepsilon\left(1-x^{2}\right) x$}

We will consider the case

$$
h:[-1,1] \rightarrow(-1,1), \quad h(x)=\varepsilon\left(1-x^{2}\right) x^{2 n+1},
$$

$n$ nonnegative integer, $\varepsilon$ small positive constant that obviously satisfies all conditions needeed, including positiv sectional curvature.

For the sake of simplicity, we will consider the case

$$
h:[-1,1] \rightarrow(-1,1), \quad h(x)=\varepsilon\left(1-x^{2}\right) x,
$$

with $0<\varepsilon<\frac{1}{2}$. Observe that for this $h$, we have

$$
h^{\prime}(x)=\varepsilon\left(1-3 x^{2}\right), \quad h^{\prime \prime}(x)=-6 \varepsilon x,
$$

and the Gauss curvature

$$
G(x)=-\frac{2 \varepsilon x^{3}+1}{\left(\varepsilon x^{3}-\varepsilon x-1\right)^{3}}
$$


of $(\Lambda, g)$ is a smooth function, with only one real zero at $x=-\frac{\sqrt[3]{4 \varepsilon^{2}}}{2 \varepsilon}$ taking the value 1 at $x=0$, and $G(-1)=1-2 \varepsilon$, that is $G>0$ for any $x \in[-1,1]$ and any $\varepsilon<\frac{1}{2}$.

In this case from Theorem 6.2 we have

$$
\frac{1-v_{1}^{2}}{\cos ^{2} R}=\left(-v_{2}+\varepsilon v_{1}^{2} \cos ^{2} R-\varepsilon c^{2}\right)^{2} .
$$

For obtaining the algebraic equation in $F$, the fundamental function of this Finsler space, we simply substitute $v_{i}$ by $\frac{v_{i}}{F}, i=1,2$ since this is the indicatrix equation where $F=1$. It follows

$$
\frac{F^{2}-v_{1}^{2}}{\cos ^{2} R}=\frac{\left(\varepsilon c^{2} F^{2}-\varepsilon v_{1}^{2} \cos ^{2} R+F v_{2}\right)^{2}}{F^{2}},
$$

and from here we obtain the 4 -th order equation in $F$, whose solution gives the explicit form of the desired Finsler metric. Since the formulas are quite complicated, instead of writing them explicitely here (this can be done very easily) with the substitution $c=\sin R$, the implicit equation 6.10 can be used to obtain some graphical representations of the indicatrices for different values of the parameter $\varepsilon$ at different points of $M$.

The invariants $I$ and $J$ of this Finsler surface can be easily be obtained using (6.9) and 2.14 . 

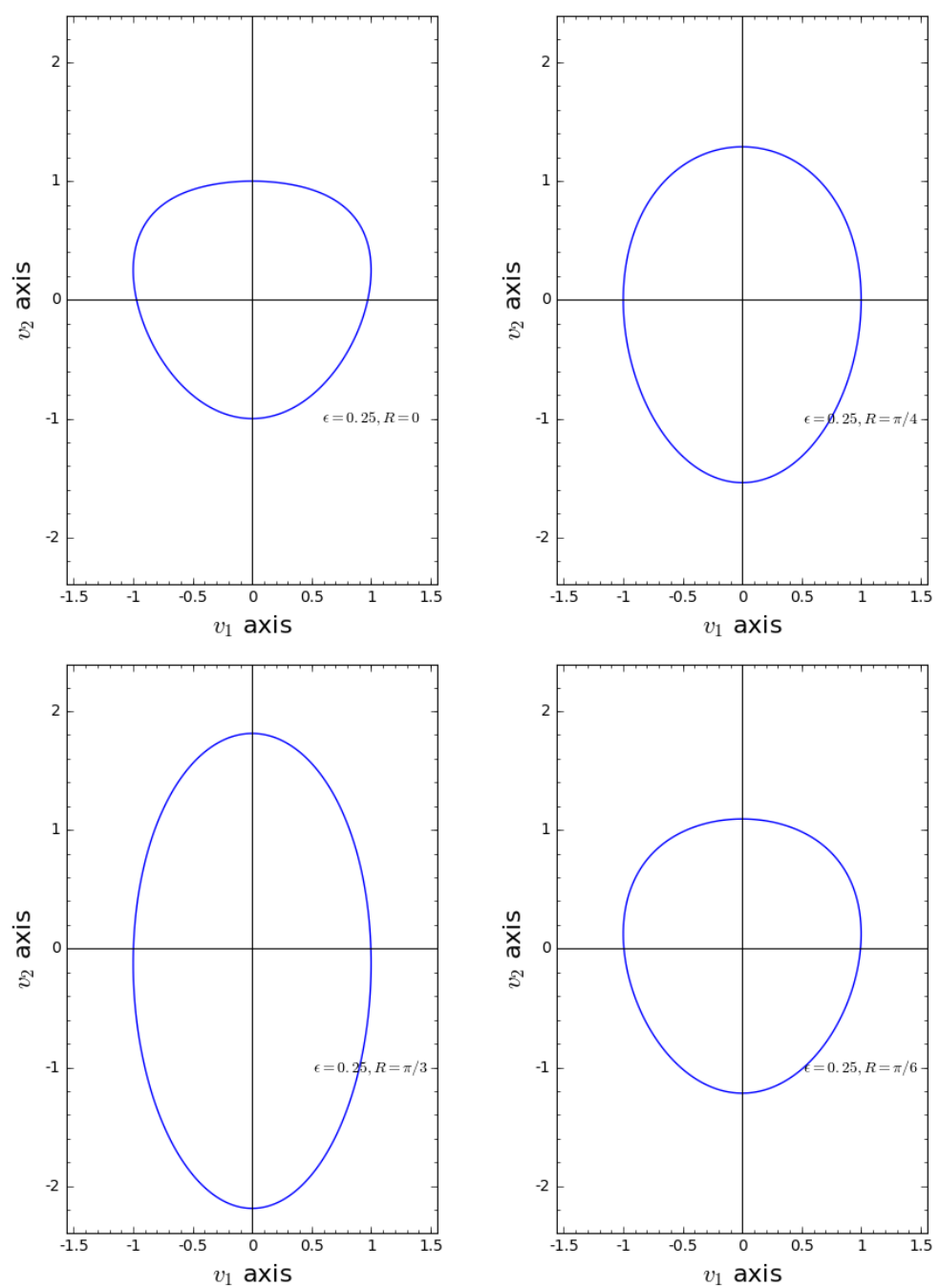

Figure 9: Indicatrices in Example 1 for $\varepsilon=0.25$. 

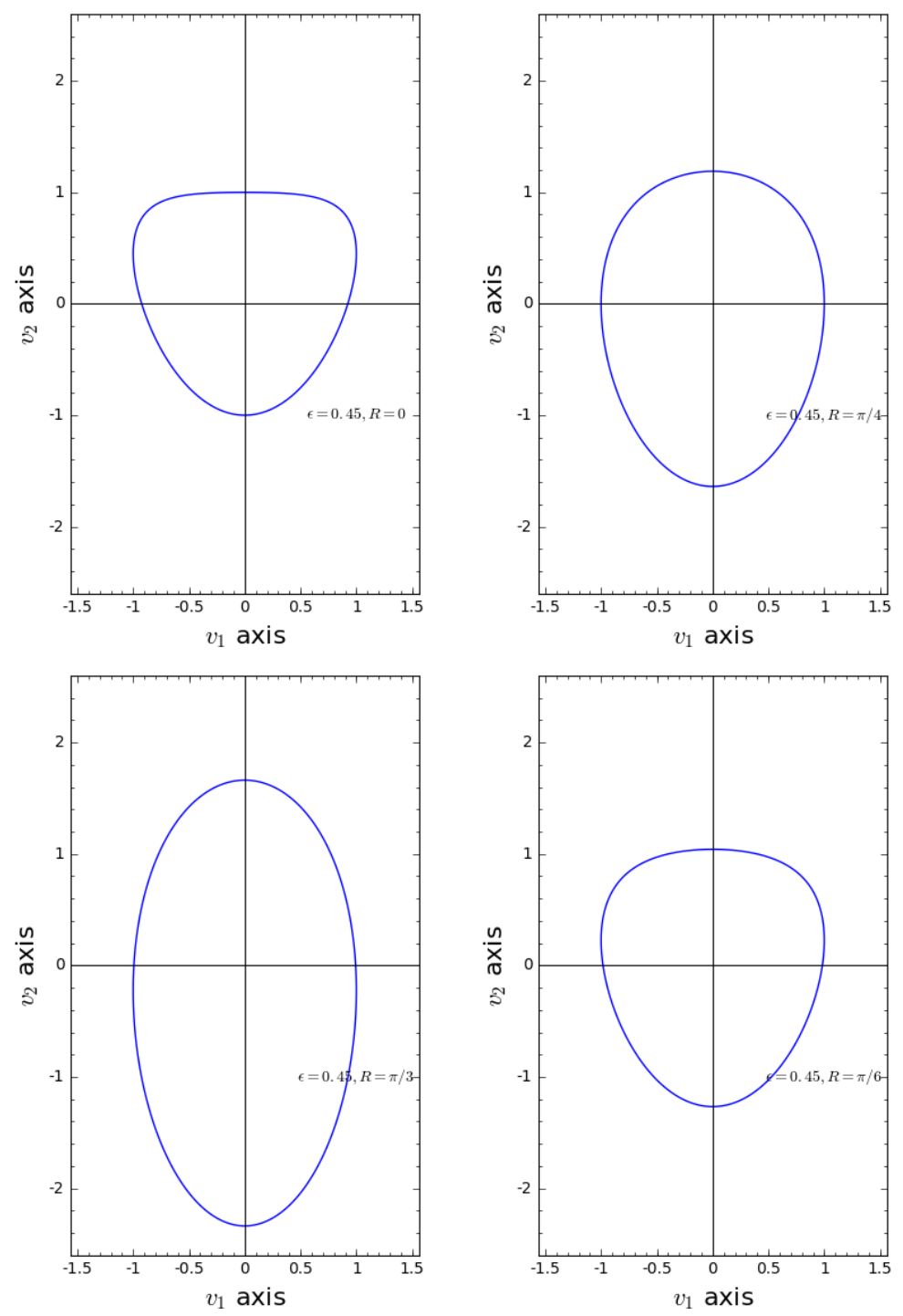

Figure 10: Indicatrices in Example 1 for $\varepsilon=0.45$.

\subsection{Example 2. The case $h(x)=x\left(1-x^{2}\right)^{2}$}

Another possible choice is the case

$$
h:[-1,1] \rightarrow(-1,1), \quad h(x)=x\left(1-x^{2}\right)^{n},
$$

$n>1$ integer that obviously satisfies all conditions needeed, including positiv sectional curvature. For the sake of simplicity, we will consider the case

$$
h:[-1,1] \rightarrow(-1,1), \quad h(x)=x\left(1-x^{2}\right)^{2} .
$$

Observe that for this $h$, we have

$$
h^{\prime}(x)=1-6 x^{2}+5 x^{4}, \quad h^{\prime \prime}(x)=-12 x+20 x^{3},
$$


and the Gauss curvature

$$
G(x)=\frac{1+h(x)-x h^{\prime}(x)}{[1+h(x)]^{3}}=\frac{1+4 x^{3}-4 x^{5}}{\left(1+x-2 x^{3}+x^{5}\right)^{3}} .
$$

It can be easily seen that $G(x)>0$ for $x \in[-1,1]$. Indeed, observe that $G(-1)=$ $G(1)=1$, and that the critical points and the critical values of the function $G:[-1,1] \rightarrow$ $\mathbb{R}$ are

$$
\begin{aligned}
& x_{0}=0.33, \quad G\left(x_{0}\right)=0.56 \\
& x_{1}=0.88, \quad G\left(x_{1}\right)=1.42 \\
& x_{2}=-0.35, \quad G\left(x_{2}\right)=2.18 \\
& x_{3}=-0.81, \quad G\left(x_{3}\right)=0.36,
\end{aligned}
$$

and since $G$ is continuous function it follows that cannot take nonpositive values.

In this case from Theorem 6.2 we have

$$
\frac{1-v_{1}^{2}}{\cos ^{2} R}=\frac{1}{9}\left[v_{1}^{4} \cos ^{4} R+6 v_{1}^{2} \cos ^{2} R \sin ^{2} R-3 \sin ^{4} R-3 v_{2}\right]^{2}
$$

that leads to a 8-th order polynomial in $F$.

Again, the invariants $I$ and $J$ of this Finsler surface can be easily be obtained using 6.12) and (2.14).

\section{References}

[1] D. Bao, S.S. Chern, Z. Shen, An Introduction to Riemann-Finsler Geometry, Springer, GTM 200, 2000.

[2] Bryant, R., Finsler structures on the 2-sphere satisfying $K=1$, Finsler Geometry, Contemporary Mathematics 196 (1996), 27-41.

[3] Bryant, R., Some remarks on Finsler manifolds with constant flag curvature, Houston Journal of Mathematics, vol. 28, no.2 (2002), 221-262.

[4] Besse, A., Manifolds all of whose geodesics are closed, Springer-Verlag, 1978.

[5] Kiyohara, K., Compact Liouville Surfaces, J. Math. Soc. Japan, Volume 43, Number 3 (1991), 555-591.

[6] LeBrun, C., Mason, J. L., Zoll manifolds and complex surfaces, J. Diff. Geometry, 61 (2002), 453-535.

[7] Matveev, V., Shevchishin, V., Differential invariants for cubic integrals of geodesic flows on surfaces, J. Geom. Phys. 60(2010) no. 6-8, 833-856.

[8] Sabau, S. V., Shibuya, K., Shimada, H., Moving frames on generalized Finsler structures, J. Korean Math. Soc. 49 (2012), no. 6, 12291257.

[9] Sabau, S. V., Shibuya, K., Pitis, Gh., Generalized Finsler structures on closed 3-manifolds, Tohoku Math. J., vol. 66, no. 3 (2014), 321-353. 